\title{
Study on the effect of geophysical blasting vibration on buildings
}

\author{
Qi Jingjing ${ }^{1, a}$, Yin Zhiqing ${ }^{1, b}$, Wu Kongbing ${ }^{1, c}$ and Liu Fuxing ${ }^{1, d}$ \\ ${ }^{1}$ Shengli Oilfield Technology Inspecrtion Center, SINOPEC; Shandong Dongying, China \\ aqijingjing533.slyt@sinopec.com, byinzhiqing685.slyt@sinopec.com, \\ cwukongbing.slyt@sinopec.com
}

Keywords: geophysical blast ; vibration effect ; vibration wave

\begin{abstract}
In the process of oil field exploration and development, the vibration induced by geophysical blast, drilling construction and overhaul operations, etc, are often encountered. The vibration have a certain impact on the surrounding houses or poultry and livestock farmers. And the normal operation of oil field exploration and development is affected by the dispute of worker and peasant. The ground vibration caused by the geophysical blast has great influence on the surrounding environment, so the effect of geophysical blasting vibration on building is studied in this paper. Based on the dynamic method, the dynamic response process of building structure to blasting vibration is analyzed. And the effects of vibration amplitude, frequency and duration on the dynamic response of building structures are also studied in this paper. The research results have important significance to the oil field exploration and development.
\end{abstract}

\section{Introduction}

Geophysical blast is the main work of finding oil in the oil field. The main work is to achieve the purpose of looking for oil storage structure by obtaining the propagation law of vibration wave with different depth and different charge quantity. Its work area is very wide, not only to be implemented in the suburbs, but also often through the city. So it puts forward higher request to the vibration caused by the geophysical blast. On the one hand, the technical requirements of geophysical work must be met, on the other hand, it is also be ensured that the ground buildings cannot be damaged in the blasting process. So the influence of characteristics of vibration wave, blasting vibration effect and blasting seismic wave parameters $\mathbf{I}^{1} \sim^{3} \mathbb{I}$ caused by geophysical blast on the buildings is studied comprehensively.

\section{Characteristic analysis of vibration wave induced by geophysical blast}

Because of the complexity of the blasting vibration wave, the variety of the physical and mechanical properties of the propagation medium, and the variety of the geological structure, the blasting vibration wave has the random and non repetitive characteristics. The amplitude of blasting vibration changes complexly with time, and the wave spectrum and duration also change complexly with blasting environment conditions, the distance from the explosion source, the maximum single ring, the total amount of charge and the geological conditions $\mathbf{I}^{4} \sim 5 \mathbf{I}$.

Energy propagation characteristics of blasting vibration wave. Compared with the vibration wave caused by the earth's crust movement, the blasting vibration wave has the characteristics of abundant frequency, concentrated frequency band and characteristics of transient shock vibration. And the propagation distance of low frequency blasting vibration wave is greater. The high frequency components are rich and the duration is short near the explosive source. In the greater distance, the particle vibration velocity amplitude becomes smaller, the high frequency components attenuate faster and the low frequency component of the main vibration frequency increased considerably.

The richness of spectrum characteristics of blasting vibration wave. The spectrum characteristics reflect the change rate of blasting vibration wave. The change rate of the high frequency wave is faster, and the change rate of the low frequency wave is relatively slow. The frequency spectrum of blasting 
vibration wave is very rich, which contains all of the frequency, from $0 \mathrm{~Hz}$ to infinity $\mathrm{Hz}$. And the frequency spectrum is a continuous spectrum, but not discrete frequency components.

Wave characteristics of blasting vibration wave. The propagation of blasting vibration wave in rock and soil medium is the synthesis of complex wave process. The wave spread from the explosive source to the surrounding through the medium. Because the spread velocity of longitudinal wave, shear wave and surface wave are different, the epigenetic wave, for example the reflection wave and the refraction wave, are produced continuously in rock and soil medium. The epigenetic wave and the direct transmission wave continuously add and subtract, which induce the waveform parameters(amplitude, frequency, and phase, etc.) of blasting vibration wave change continuously with time.

Mechanism of blasting vibration wave. According to the effect of blasting vibration wave on the building structure, the damage mechanism can be divided into two kinds: the mechanical effect of vibration wave and the strain effect of vibration wave.

The mechanical effect of blasting vibration wave is reflected in the tension and pressure acting directly on the structure. The effect is similar to the effect of the overpressure and impulse of the air shock wave on the structure. The following formula can be used to solve:

$$
m(t)+\rho_{0} c_{0} s X(t)+c X(t)=2 s \Delta P(t)
$$

Here, $s$ is the area under the overpressure of the structural surface; $m$ is quality; $\rho_{0} c_{0}$ is the wave

impedance of medium; $c$ is stiffness coefficient; $t)$ ( $\mathcal{C}_{(t)}$ and $X(t)$ are respectively the acceleration, speed, and displacement of the structure.

The strain effect of vibration wave is shown as the structural deflection, which is induce by the transferring of vibration from medium to structure. It can be divided in to two types, one is produced by the influence of the inertia effect, the other is produced by the combined action of the inertia force and relative displacement of different structure under the action of blasting vibration.

\section{Dynamic response analysis of blasting vibration wave in building}

Blasting vibration effect. Blasting vibration effect ${ }^{[6} \sim^{7} I$ means the damage to different degrees of buildings induced by the seismic waves. The seismic waves are converted by a part of the blasting energy. And the building is not the blasting target. It is assumed that the medium is homogeneous and isotropic elastic body, and the medium particle vibrates with simple harmonic motion. The state of motion of the medium particle can be described by the displacement $\mathrm{X}$, the velocity $\mathrm{V}$, and the acceleration a:

$$
X=A \sin \omega t
$$

2)

$$
v=\frac{d x}{d t}=\omega A \sin \left(\omega t+\frac{\pi}{2}\right)
$$

3)

$$
a=\frac{d V}{d t}=\omega^{2} A \sin (\omega t+\pi)
$$

Here, $\mathrm{t}$ is vibration time; $\mathrm{A}$ is the maximum amplitude; $\omega$ is the circle frequency.

The measured data show that it is more favorable to use the particle vibration velocity as the standard to measure and describe the blasting vibration intensity. Because the particle vibration velocity can eliminate the influence of rock mass medium. Moreover, the vibration velocity of a particle is a relatively stable physical quality, which does not change with the change of rock mass property. If we assume the vibration of the rock mass as a simple harmonic motion, then the vibration velocity of the vibration particle can be expressed as the following form: 
$v=\frac{2 \pi A}{T}$

5 )

Here, $\mathrm{A}$ is the displacement amplitude of particle vibraton; $\mathrm{T}$ is the vibration period; $\mathrm{f}$ is the frequency.

When the building is subjected to vibration, therer is a relationship between the strain $\varepsilon$, elastic modulus $\mathrm{E}$ and stress $\sigma$ :

$$
\sigma=E \varepsilon
$$

)

Based on the above two formula, the relationship between the stress $\sigma_{m}$ and the ultimate velocity $v_{m}$ is obtained:

$$
\sigma_{m}=E v_{m} / c
$$

)

It is concluded that the stress caused by blasting vibration in building is proportional to the vibration velocity of rock mass media. And the vibration velocity of rock mass media is one of the important physical quantities to determine the damage of buildings.

Dynamic response of buildings to blasting vibration wave. The building can be simplified as a single degree of freedom system, if the seismic wave is regarded as a simple harmonic wave. The mass of a single degree of freedom elastic system is $\mathrm{m}$, the elastic constant is $\mathrm{k}$, the dampling coeffient is $\mathrm{c}$, $\mathrm{P}(\mathrm{t})$ is the varying load changing with time

The ground vibraition law is :

$$
v(t)=v_{\max } \sin \omega t
$$

\section{8 )}

Here, $\omega$ is the frequency of the external load, $v_{\max }$ is the maximum displacement of the ground vibration.

Corresponding to the formula(8), the seismic accerleration and seismic force are:

$$
=-\omega^{2} v_{\max } \sin \omega t
$$

9)

$$
P(t)=-m(t)=-m \omega^{2} v_{\text {max }} \sin \omega t=-P_{0} \sin \omega t
$$

10 )

The motion eqation of the building is :

$$
\left.+2 \xi \omega_{0}\right)(t)+\omega_{0}^{2} v=-\frac{P_{0}}{m} \sin \omega t
$$

)

Here, the $P_{0}$ is the maximum value of the load; $\omega_{0}$ is the natural frequency of the building, $\omega_{0}$ is the equivalent viscous damping retio.

The general solution for equation ( 11$)$ is :

$$
v(t)=e^{-\xi \omega t}\left(A \cos \omega_{D} t+B \cos \omega_{D} t\right)+\frac{P_{0}}{k}\left[\left(1-\beta^{2}\right)+(2 \xi \beta)^{2}\right]^{-1} \cdot\left[\left(1-\beta^{2}\right) \sin \omega t-2 \xi \beta \cos \omega t\right]
$$

\section{The influence of blasting vibration wave parameters on buildings.}

The influence of seismic amplitude value on dynamic response of buildings. The first term in the equation (12) indicates the transient response of the acting load, and this term is not significant. The 
second term in the equation is the steady-state response of the acting, which has the same frequency and different phases.

Composite vector $\mathrm{p}$ represents the amplitude of the steady state response:

$$
p=\frac{P_{0}}{k}\left[\left(1-\beta^{2}\right)^{2}+(2 \xi \beta)^{2}\right]^{-0.5}
$$

)

For the above formula, in the case of other conditions unchanged, the relative amplitude of the steady-state response of the building will depend on the maximum amplitude of the blasting vibration. So it can be concluded that the amplitude of blasting seismic wave has a great influence to the building. The influence of seismic frequency on dynamic response of buildings. If $p$ is the amplitude of buildings under the earthquake load, $p_{0}$ is the amplitude of the buildings under the static load, then the dynamic magnification coefficient $\mathrm{D}$ can be expressed as follows:

$$
D=\frac{p}{P_{0} / k}=\left[\left(1-\beta^{2}\right)^{2}+(2 \xi \beta)^{2}\right]^{-0.5}
$$

Here, is the ration of ground vibration frequency to natural frequency of buildings.

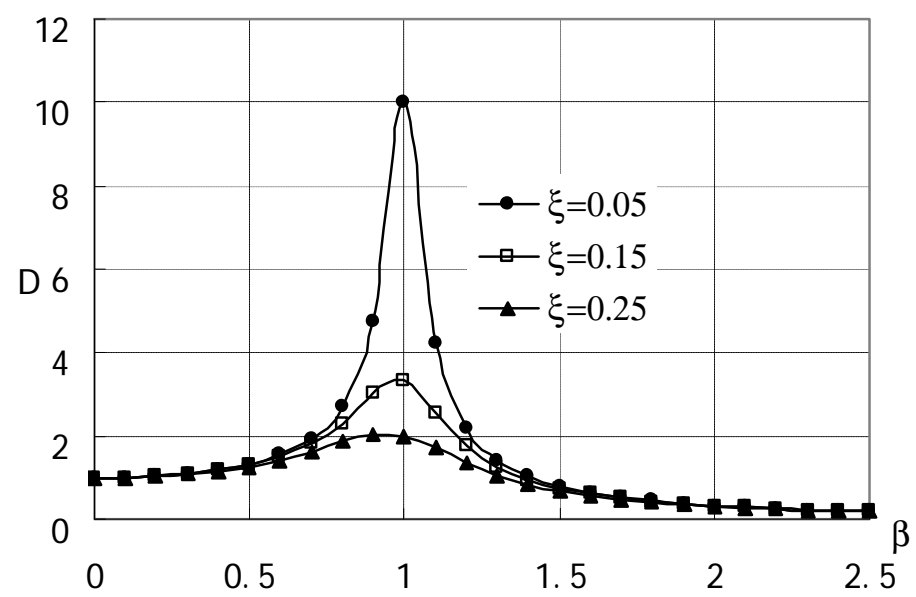

Fig,1 Relationship between dynamic amplification factor and frequency ratio

The relationship between the dynamic amplification coefficient $\mathrm{D}$ and the frequency ratio is as shown in Figure 1. It can be seen from the figure that when the frequency $\beta=\omega / \omega_{0}$ approach in $\infty$

, $\mathrm{D}$ tends to 0 . This indicates that under the action of high frequency seismic, amplitude is very small.

When the frequency $\beta=\omega / \omega_{0}$ approach in 1 , D increases very fast. Then the resonance phenomenon occurs, and the building amplitude reahces the maximum value. The maximum value of the amplification factor is greatly affected by the daming ratio. The greater the damping ration, the smaller the dynamic magnification factor.

The influence of the seismic wave duration on the dynamic response of building. The duration of balsting vibration is very short, generally in $0.5-2.5 \mathrm{~s}$. This duration can increase the damage accumulation of the inelastic deformation of the building. Based on the analysis of the mechanism of structural damage, this is a process from the nonlinear deformation to the complete collapse of the structure. The structural deformation often have to go through a number of reciprocating vibration process. The non recovery of plastic deformation requires the energy dissipation. So in the process of vibration, even the structural deformation does not reach the maximum deformation under the static condition, the structure may also reaches a certain limit and collapses because of the energy depletion. The damage is called cumulative damage. 
The harm of seismic duration to buildings also reflect on the resonance. In fact, even if the vibration frequency is exactly the same with the self vibration frequency, the single vibration will not cause resonance.

\section{Conclusions.}

In this paper, the dynamic response of buildings to blasting vibration by dynamic method is studied. The influence of vibration amplitude, frequency and duration on the dynamic response of building is shown in this paper. Research shows as follows:

The amplitude of blasting vibration wave is very big to the building.

For the vibration load with the same direction and size, different vibration frequency, the response of structure is different. The influence of vibration load close to the natural frequency of the structure is larger than that of the other frequency range. The damage effect of blasting vibration duration is mainly manifested in the structural response of nonlinear process. Because of the increase of vibration time, the loss of strength is induced when the structural response is beyond the elastic limit. The damage effect of blasting seismic wave duration on building is also reflected in the resonance.

\section{References}

[1] Lu Wen-bo, Zhang Le, Zhou Jun-ru, et al: Theoretical analysis on decay mechanism and law of blasting vibration frequency, BLASTING, Vol. 30 (2013), p. 1-6.

[2] Zhang Li-guo, Gong Min, Yu Ya-lun: Forecast and regression analysis of blasting vibration frequency, Journal of Liaoning Technical University, Vol. 4 (2005), p. 187-189.

[3] Gao Fu-qiang, Hou Ai-jun, Yang Xiao-lin, et al. Analysis of blasting vibration frequency based on dimensional method, BLASTING, Vol. 27 (2010), p. 1-3.

[4] Meng Hai-li, Guo Feng: Experimental research on the master frequency of blasting seismic wave, Journal of Railway Engineering Society, Vol.11(2009), P.81-83.

[5] Fotis, Sambuelli L, Comina C, et al: The role of surface waves in prediction of ground vibration from blasting. Spathis \& Noy(eds), London: Taylor \& Francis Group. (2010)

[6] Fu Shi-gen, Wang Yun-hai, Xu Kai-li: Study on the evaluation of blast vibration effect and measures of reducing vibration, Journal of Safety Science and Technology, vol.4(2008). P. 25-28.

[7] Wu De-lun, Ye Xiao-ming: A comprehensive review and commendation of blast vibration safety velocity, Chinese Journal of Rock Mechanics and Engineering, vol.16(1997), P.266-273. 